\title{
Projeto de um Controlador Fuzzy Aplicado em um Tanque de Nível de uma Planta didática de Processos Industriais
}

\author{
Francisco Leão de Oliveria* José Medeiros de Araújo Junior* \\ Pedro Iago Carvalho Martins* \\ Lindokeny Rodrigues de Sousa Moura* \\ * Programa de Pós-Graduação em Engenharia Elétrica, Universidade \\ Federal do Piauí, PI \\ e-mail: fco.leao@hotmail.com,jmjunior@ufpi.edu.br, \\ pjago@protonmail.com, lindokeny@gmail.com.
}

\begin{abstract}
In this work a study and the implementation of level control is presented, using the MPS-PA Festo industrial process module. This process has non-linear characteristics, and it was initially analysed in closed-loop, where, after watching its response, it was developed via MATLAB a fuzzy controller. To test the performance of the fuzzy controller, it was compared with a PI controller. The experimental results show a basis for comparation between the two control techniques.

Resumo: Neste trabalho é apresentado um estudo e implementação do controle de nível no modulo didático de processos industriais MPS-PA Festo. Este processo, que apresenta características não lineares foi analisado inicialmente em malha fechada, no qual, após um estudo das características de sua resposta, implementou-se, via MATLAB um controlador fuzzy. Para avaliar o desempenho do controlador fuzzy, será realizada uma comparação com o controlador PI. Os resultados obtidos experimentalmente apresentam uma base para a comparação entre as duas técnicas de controle.
\end{abstract}

Keywords: Level Control; Fuzzy Control; PI Control; MSP-PA Plant.

Palavras-chaves: Controle de Nível; Controle Fuzzy; Controle PI; Planta MSP-PA;

\section{INTRODUÇÃO}

As indústrias estão procurando cada vez mais otimizar a dinâmica de seus processos. Entre os vários processos encontrados, uns dos mais conhecidos são os tanques de nível, utilizados para armazenamento de fluídos, e presentes nas indústrias farmacêuticas, de alimentos, químicas, etc (Prusty et al., 2014). Na busca pela otimização desses sistemas, recorre-se normalmente ao uso de controladores, que são responsáveis por manter os diferentes pontos de ajuste de nível em um tanque, fazendo com que os mesmos apresentem uma resposta satisfatória (Parvat and Patre, 2015).

$\mathrm{Na}$ literatura são encontrados diversos trabalhos sobre controle de tanques de nível. Franzini et al. (2013) para realizar um controle em tanques acoplados apresentaram a comunicação de instrumentos de uma planta didática, através do padrão OPC (OLE for Process Control), amplamente usado em ambiente industriais, e com programação através de software Matlab/Simulink, sendo assim uma solução pratica para troca de informações em sistemas de controle reais.

Embora tradicionalmente os trabalhos de controle de nível façam o uso de controladores PID (Kannan et al., 2010), é possível encontrar também muitos trabalhos envolvendo controladores fuzzy (Shaw, 2007). Esses controladores são baseados em lógica de múltiplos valores, o algoritmo de processo é aproximado, ou seja, não é fixo. O conhecimento e a experiência do especialista são convertidos em um nível de máquina facilmente com a ajuda de regras e, em seguida, colocados em uma base de regras do controlador para tomar as decisões adequadas. Portanto, a implementação do controlador torna-se fácil e pode ser obtida sem modelagem matemática complexa (Lamkhade et al., 2015). Trabalhos como o de Bhandare and Kulkarni (2015) ressaltam que o controlador Fuzzy é usado para obter uma boa resposta em sistemas reais de natureza nãolineares, respostas essas que controladores convencionais nem sempre são capazes de fornecer. Guarnizo and Avendano (2017) ao implementar um controle de nível utilizando lógica fuzzy, concluiu que essa é uma boa alternativa para controle de níveis, pois o mesmo é de natureza não linear.

Em termos acadêmicos, técnicas de controle de nível podem ser aplicadas em plantas didáticas, como a planta MPS-PA da Festo, que fornece a possibilidade de trabalho com quatro sistemas utilizados em processos industriais como: o controle de nível, o controle de temperatura, a vazão de uma tubulação e a pressão exercida por um líquido. Os sistemas podem ser manuseadas individualmente ou de uma forma conjunta, formando uma planta industrial com- 
plexa (Silva et al., 2014). Utilizando a comunicação OPC é possível estabelecer uma comunicação com a planta, projetando assim um controle de nível para esta.

Neste contexto, esse trabalho propõem um projeto de um controlador fuzzy para controlar o nível de água de um tanque didático MSP-PA Festo. Além disso, para fins de comparação com um controle convencional, o controlador PI também é projetado para o mesmo sistema.

\section{REFERENCIAL TEÓRICO}

Nesta seção são descritas as principais características do sistema a ser controlado, juntamente com os principais conceitos necessários para implementar um controlador baseado na lógica fuzzy.

\subsection{Planta Didática MPS-PA Festo}

A planta MPS-PA da Festo é um sistema de aprendizagem didática utilizado para processos de tecnologia de automação e é determinado para atender uma série de elementos de diferentes requisitos de formação profissional e de capacitação (Helmich).

Com a planta MSP-PA é possível usar quatro sistemas de controle, são eles: temperatura, pressão, nível e vasão. O sistema utilizado nesse trabalho será o de processo do nível Figura 1. O processo de controle de nível é realizado pela bomba P101 que flui o líquido do tanque B101 para tanque B102 e o sensor ultrassônico LIC B101 localizado na parte superior do tanque B102 detecta o nível do líquido. A medição do líquido é feita no tanque B102 e a atuação do controle é a tensão aplicada na bomba. Os tanques são retangulares e possuem capacidade de 10 Litros.

A comunicação com a bancada MPS-PA é feita através do dispositivo de aquisição de dados Easyport, que permite o controle de processos via computador através do software Matlab. O Easyport possui 16 canais digitais, sendo 8 entradas e 8 saídas, bem como 4 entradas analógicas e 2 saídas analógicas (Helmich).

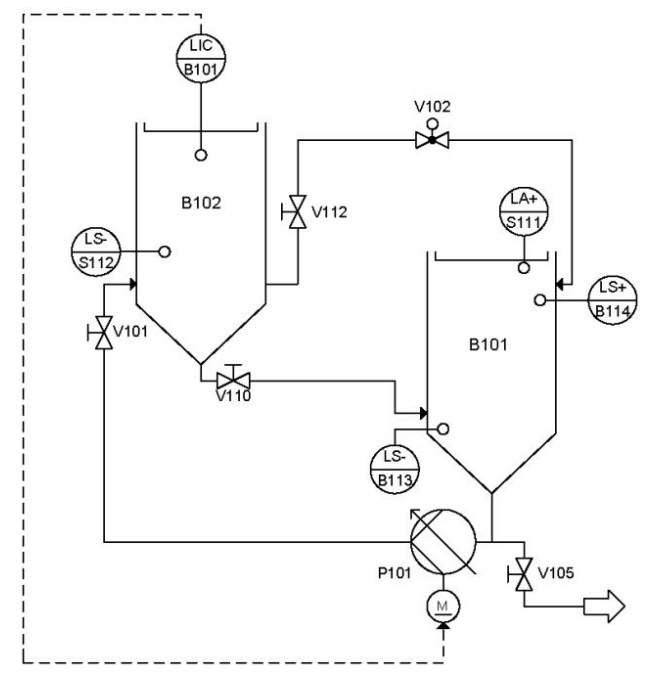

Figura 1. Sistema do Processo de Nível.

Os principais componentes e acessórios da planta MPS-PA da Festo são identificados na Figura 2 .

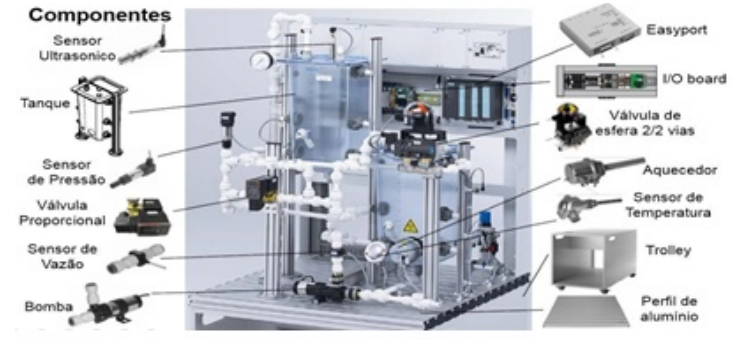

Figura 2. Bancada didática MPS-PA - Estação Compacta.

\subsection{Controle Fuzzy}

O controle fuzzy é baseado na lógica proposta por Zadeh (1965) e sua principal característica é o fato dele ser modelado a partir de um conhecimento de um especialista e não do processo em si. Comparado com os métodos tradicionais de controle que são baseados em modelagens matemáticas dos processos de modo a derivar as ações de controle, o controlador fuzzy aborda casos onde o conhecimento do especialista de controle será disponível, seja por meio de operadores ou de projetistas, principalmente em casos onde os modelos matemáticos envolvidos são muito custosos e complicados para serem desenvolvidos (Gomide et al., 1995).

Na Figura 3 é apresentada a arquitetura básica que compõe um controlador fuzzy, além de apresentar o sentido do fluxo da informação, desde a entrada dos dados no sistema até a tomada e decisão (Shaw, 2007).

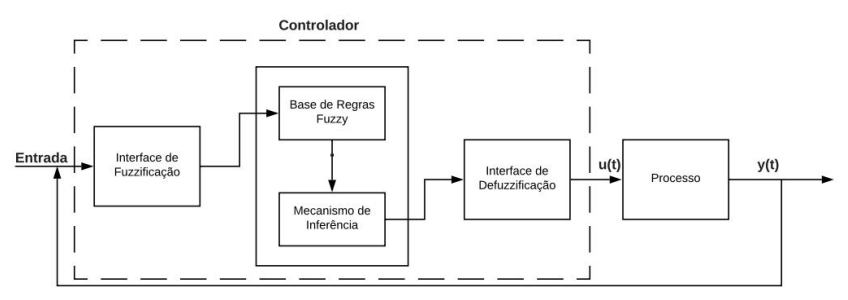

Figura 3. Arquitetura do Controlador Fuzzy.

A interface de "fuzificacão" identifica os valores das variáveis de entrada que caracterizam o estado do sistema (variáveis de estado), e as normaliza em um universo de discurso padronizado. Estes valores são então "fuzificados", com a transformacão da entrada em conjuntos nebulosos para que possam se tornar instâncias de variáveis linguísticas (Sandri and Correa, 1999).

A base de regras fuzzy consiste em um conjunto de regras para controlar o sistema. Já o mecanismo de inferência avalia quais regras de controle são relevantes no momento atual e decide qual deve ser a entrada para a planta (Lamkhade et al., 2015).

A interface de defuzzyficação transforma a região fuzzy de saída em uma resposta não fuzzy, pertencente ao universo de discurso. Os principais métodos de defuzzyficação são: o centro de área; a média dos máximos; o do primeiro máximo e o do último máximo. Dentre estes métodos, o mais utilizado é o de centro de área (Branco, 2009). 


\section{METODOLOGIA}

Para implementação do controle foi necessario o desenvolvimento da interface de comunicação entre o computador e a planta MSP-PA. Para esta etapa a comunicação foi feita por meio da interface EasyPort da Festo a qual apresentada no modelo mostrado na Figura 4. Para esta etapa empregou-se o protocolo OPC de comunicação via MATLAB.

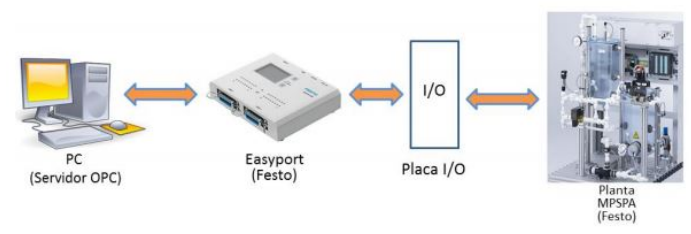

Figura 4. Modelo de Controle e Supervisão da Estação MPS PA via Easyport.

Com o ambiente e comunicação com a planta estabelecidos, determinou-se as etapas práticas deste trabalho. A primeira etapa constituiu-se em estudar por meio de testes simples aplicado ao sistema de nível da planta em malha fechada, para análise da dinâmica do sistema.

A primeira etapa de testes permitiu a estimação da função de transferência que modela o sistema. Devido o processo de controle de nível não ter caráter linear, pois a vazão está relacionada ao quadrado da altura do nível de água, ao longo de todos os experimentos a válvula V110 permaneceu com uma abertura constante.

\subsection{Ensaio em malha fechada}

Inicialmente, realizou-se o ensaio em malha fechada, com o ambiente de supervisão e controle do sensor de nível e da bomba, respectivamente, ocorrendo no simulink, como ilustrado na Figura 5. Foi escolhido como referência de entrada 4 litros. Pode-se observar que a bomba não levou o nível do tanque ao valor selecionado de referência. A medida que a saída do processo (nível do tanque) aumenta devido a ação do atuador (tensão na bomba), o valor do erro (referencia - saída) decresce, diminuindo assim a intensidade do atuador no processo, ou seja, o valor da tensão submetida a bomba diminui.

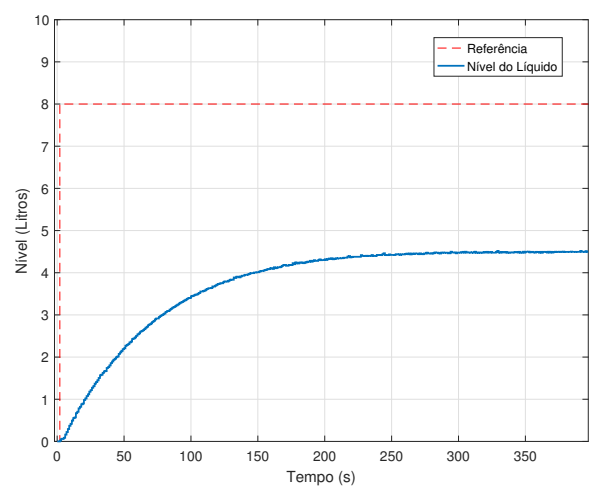

Figura 5. Ensaio em malha fechada.

O processo conta com uma válvula semiaberta abaixo do tanque (V110), para vazão de água, o que faz com que exista um fluxo de saída permanente de liquido, proporcional ao volume. Este fato faz com que a saída do processo não alcance o referencial de entrada e estabilizando o processo na faixa de 4,5 litros. Um erro próximo de $50 \%$.

Observa-se que sem a ação de um controle, o nível não alcança o valor desejado. Logo, é necessário implementar algum controle no processo para que o objetivo seja alcançado.

\subsection{Desenvolvimento do Sistema Fuzzy}

Para implementação do controlador Fuzzy, inicialmente delimitou-se as variáveis de entrada e saída do sistema. Para saída escolheu-se a tensão de alimentação da bomba. Já para entrada inicialmente adotou-se o erro da realimentação do processo. Posteriormente, foi selecionado outra variável de entrada, rate, que pode ser interpretado como a variação do nível. O Fuzzy utilizado no projeto foi o Mamdani e os conetivos lógicos escolhidos foram o "E"e "OU".

O controlador fuzzy apresentado possui dois tipos de entrada, uma variável de saída e 8 regras de inferência. Devese ressaltar que as variáveis de entrada, os limites, formatos das suas funções de pertinência, assim como as regras de inferência, foram definidas a partir do conhecimento prévio obtidos com o estudo da resposta em malha aberta e fechada do processo de nível da planta e experiências empíricas obtidas. As variáveis de entrada (erro, rate) e de saída (tensão de alimentação da bomba) estão ilustradas nas Figuras 6, 7 e 8 sucessivamente.

A variável de entrada erro foi projetada para abranger toda a faixa possível que o erro do processo pode assumir (universo de discurso), que vai do valor de -10 a 10, pois, o tanque possui a capacidade de 10 litros. Logo, foi pensado nas variáveis linguísticas referente a entrada erro, como: um erro positivo (PS), um erro negativo (NG) e um erro zero (ZR), conforme na Figura 6 . O erro positivo abrange aos níveis visto pelo sensor ultrassônico que estão abaixo da referência escolhida. O erro negativo abrange aos níveis que estão a cima da referência escolhida e o erro zero traz a pequena faixa onde o nível estará na referência escolhida.

Foram adotados apenas três curvas de pertinência. Tal fato ocorre devido a necessidade de agrupar o universo de valores do erro em uma pequena faixa para considerar o zero. Enquanto para os erros positivos e negativos, o universo de discurso é semelhante ao que teriam na logica clássica. O que as diferem é a pertinência das curvas de valores negativos e positivos, que tem menos "importância"(pertinência) quando mais próximas do valor zero.

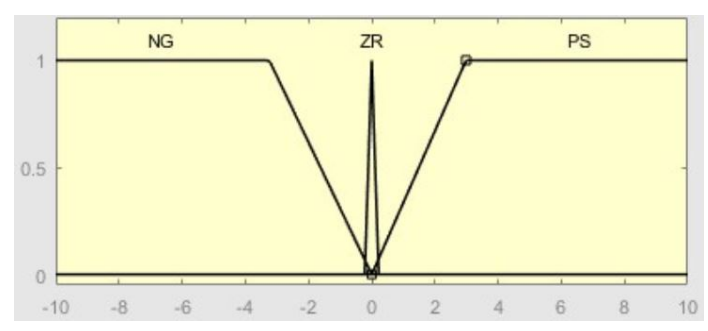

Figura 6. Função de pertinência do erro 
A função de pertinência ZR possui uma faixa mais estreita no universo de discurso para que o controlador fuzzy tenha uma resposta mais rápida.

Na variável rate, Figura 7, foram escolhidas 3 curvas, semelhantes a variável erro, no qual a curva referente o erro negativo $(\mathrm{RN})$ abrange os valores quando o nível do líquido estiver diminuindo, já a curva do erro positivo (RP) abrange os valores quando o nível do líquido estiver aumentando. A curva zero (RZ) refere-se quando o liquido estiver constante, sem variação.

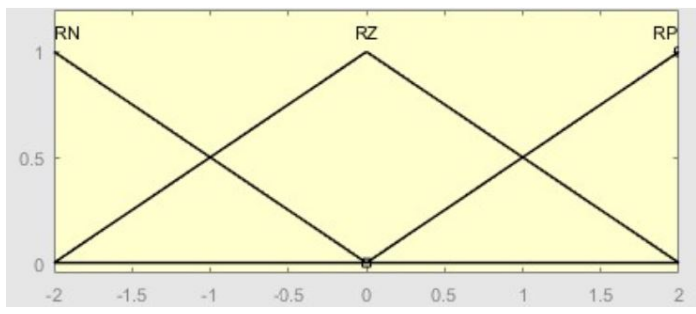

Figura 7. Função de pertinência do rate.

O alcance da entrada rate foi escolhida como sendo de -2 a 2. Se o universo de discurso fosse muito pequeno, apareceria um problema de variação na atuação do controle. Pois, a forma como é realizada a leitura do nível irá implicar em oscilações na variação do erro. Um exemplo seria quando o nível se aproximasse da referência. Neste caso, a curva de pertinência do erro seria ZR, como essa curva é um triângulo estreito (de pequena faixa de alcance) o rate poderia sofrer grande influência se a mesma também tiver uma faixa de alcance pequena. Para resolver esse problema foi aumentado a faixa de alcance do rate, diminuindo a influência da variação que curva de pertinência ZR poderia causar no atuador.

A saída do sistema representado na Figura 8 consiste em 3 curvas de pertinência, definidas como alta (AL), média (ME) e baixa (BX), onde a curva média consiste no valor de tensão enviado a bomba em que o nível irá ficar praticamente constante. Para isso, foi testado visualmente diferentes valores de tensão na bomba e escolhido um que pudesse satisfazer a seguinte condição em que a entrada de água pela bomba é praticamente igual a saída de água pela válvula. $\mathrm{O}$ valor referente a alto representa o valor aplicado na bomba que seja capaz de encher o tanque, podendo ser ele até o valor máximo de tensão escolhido utilizado na bomba e baixo um valor de tensão aplicado próximo do valor zero, onde essa função será responsável pela diminuição do nível da água.

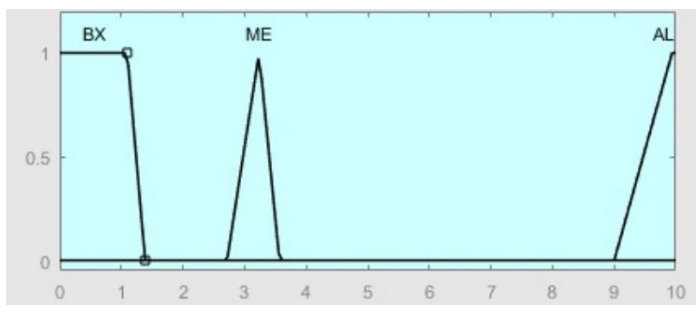

Figura 8. Tensão da Bomba.

As curvas de pertinências da saída do sistema foram definidas em três funções e estão espaçadas entre si, principalmente a função ME e a função AL. Tal fato deve-se no desejo de alcançar o sinal de referência mais rapidamente, porém a descontinuidade não gera uma mudança brusca na atuação devido a defuzzificação ser por centroide. A curva trapezoidal BX fica mais próximo de zero, por ser preciso mais "massa" para influência no centroide, diminuindo o valor da tensão aplicada na bomba, trazendo o nível para a referência quando o erro for negativo (curva de pertinência NG).

A saída do sistema, o valor da tensão do sensor de nível, é obtido por meio da defuzzificação. O método escolhido foi o Centroide, que usa o centro de massa da região fuzzy de saída. Ele utiliza a soma total de cada valor dos elementos do universo de discurso, ponderados por suas pertinências correspondentes, com esse consciente dividido pela soma de todos os elementos do universo de discurso ativos na região fuzzy de saída. Este método foi escolhido para que não ocorresse saltos na saída do controlador, instabilidade. Para esse caso, o mais indicado é a defuzzificação pelo centro de massa, pois essa possui uma maior continuidade na saída.

O controlador fuzzy foi implementado com 8 seguintes regras:

(1) $\boldsymbol{S E}($ erro É $Z R$ ) Então (Bomba É $M E)$ (0.25).

(2) $\boldsymbol{S E}$ (erro É $P S$ ) Então (Bomba É $A L)$ (0.25).

(3) $\boldsymbol{S E}$ (erro É $P S) \mathbf{E}$ (rate É RN) Então (Bomba É $A L)(1,0)$.

(4) $\boldsymbol{S E}($ erro É $N G) \mathbf{E}$ (rate É RP) Então (Bomba É $B X)(0,3)$.

(5) $\boldsymbol{S E}$ (erro É $P S) \mathbf{E}$ (rate É RZ) Então (Bomba É $A L)(1,0)$.

(6) $\boldsymbol{S E}($ erro É $N G) \mathbf{E}$ (rate É RZ) Então (Bomba É $B X)(1,0)$.

(7) $\boldsymbol{S E}$ (erro É $N G$ ) Então (Bomba É $B X)(0.25)$.

(8) $\boldsymbol{S E}($ erro É $N G) \mathbf{E}$ (rate É RN) Então (Bomba É $B X)(1,0)$.

Em muitos sistemas de nível existentes na literatura, programa-se uma válvula pneumática para controlar a vazão de escoamento do líquido. O controle de nível utilizado no presente trabalho não utilizou uma válvula modificada via software, e com isso a única variável de controle utilizada no processo foi a tensão de alimentação da bomba. A abertura da válvula de vazão da água do tanque foi escolhida no início dos estudos, mantendo-se inalterada em todos os ensaios, uma vez que sua mudança levaria a uma alteração no processo.

\subsection{Controle PI}

Apresenta-se em (1) o modelo integrador identificado para o tanque de nível em resposta ao degrau em malha aberta, por aproximação linear do mínimo erro quadrático.

$$
G(s)=\frac{8.1048 \times 10^{-3}}{s}
$$

Para efeito de comparação ao controlador fuzzy, sintonizouse o controlador PI adequado à função de malha fechada (2), de frequência natural $\omega_{n}=0.1 \mathrm{rad} / \mathrm{s}$ e fator de amortecimento $\zeta=0.6$. A escolha dos parâmetros em relação à (1) caracteriza uma resposta rápida e pouco amortecida. 


$$
G(s)=\frac{0.01}{s^{2}+0.12 s+0.01}
$$

\section{RESULTADOS}

Para avaliar e comparar o desempenho dos dois controladores, aplicou-se uma sequência idêntica de degraus. Ambos os controladores foram saturados em um valor máximo de $9.66(\mathrm{~V})$, e um valor mínimo de $0.6304(\mathrm{~V})$, para que a bomba não funcione em seu valor máximo de tensão, de $10(\mathrm{~V})$, e e nem desligue completamente.

\subsection{Controle Fuzzy do Nivel para a entrada degrau}

O comportamento do sistema controlado por fuzzy, para a sequência de degraus é apresentado na Figura 9. Observase que em todos o degraus o nível alcançou a referência sem sobressinal, e que a resposta transitória foi uma reta de inclinação correspondente à atuação limite. Devido à atuação assimétrica, com a bomba no enchimento e a válvula no escoamento, nos degraus crescentes observou-se uma inclinação maior, e então uma resposta mais rápida do que nos degraus decrescentes de mesma altura.

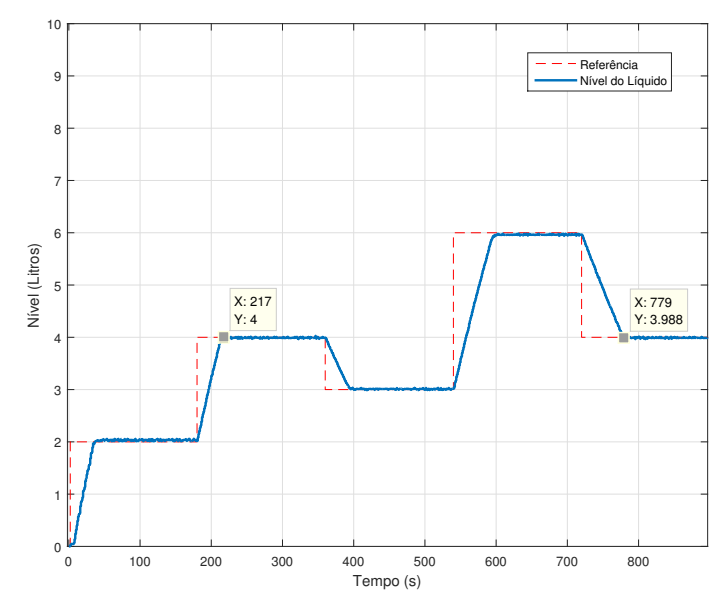

Figura 9. Sequência de degraus - Controlador Fuzzy.

Observa-se na atuação de controle fuzzy, Figura 10, que a transição entre o valor de saturação e o valor de equilíbrio é veloz e bem determinada, devido as regras e funções de pertinência da entrada erro. Em contrapartida, como a atuação de equilíbrio varia com o peso da coluna de líquido e a função de pertinência ZR tem baixa probabilidade, o controlador apresenta ruído quando próximo da referência.

\subsection{Controle PI do Nível para a entrada degrau}

Na resposta do controlador PI observada na Figura 11, por construção, observa-se um tempo de resposta próximo ao do controle fuzzy. Em teoria, parametrizando-o com uma frequência natural maior, o controle PI seria ainda mais rápido. Entretanto, um aumento da frequência natural implicaria em um aumento do ganho proporcional, e portanto piorando o transitório. Dessa forma, para se equiparar ao tempo de resposta do fuzzy houve um compromisso de escolha entre a frequência natural e o coeficiente de

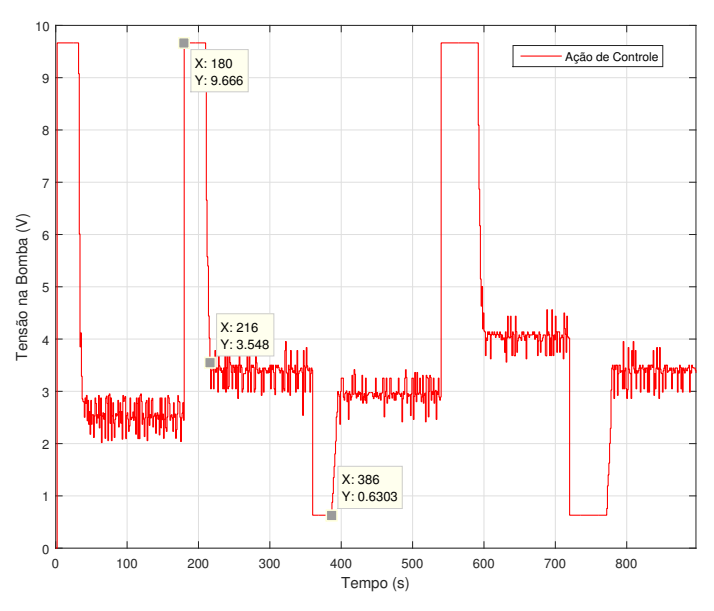

Figura 10. Ação do Controlador Fuzzy.

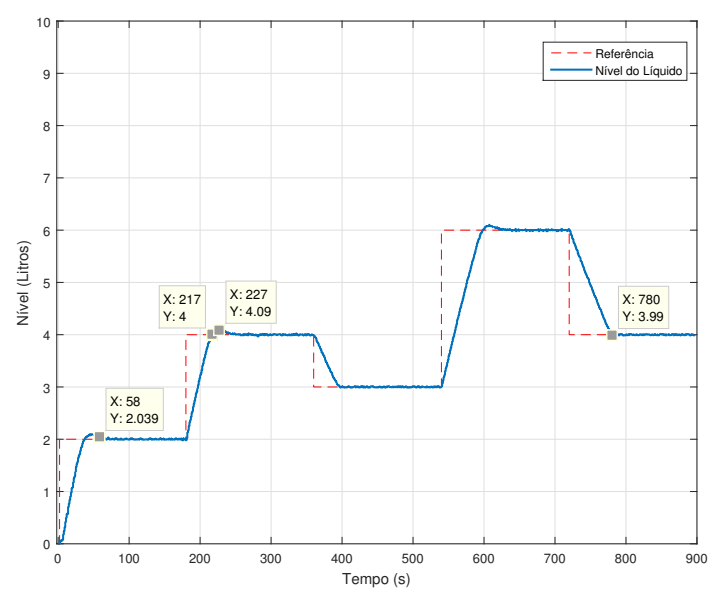

Figura 11. Sequência de degraus - Controlador PI.

amortecimento. Com os parâmetros obtidos, o sobressinal gerado foi próximo à $5 \%$.

$\mathrm{Na}$ atuação do controlador PI, Figura 12, nota-se que a transição entre a saturação e o valor de equilíbrio é mais suave do que a do controlador fuzzy. Em contrapartida, esse tempo maior de transição é o que provoca o overshoot na saída. Em tanques de nível com área de secção considerável, um pequeno overshoot de altura já provoca um grande deslocamento de volume de líquido. Nesse sentido, a necessidade de eliminar o overshoot se torna mais relevante.

Na Figura 13 mostra-se o erro dos controladores, e nota-se que o controlador fuzzy elimina o erro sem a necessidade de sobressinal, ao contrário da resposta do controlador PI. Devido a saturação, tanto o fuzzy quanto o do PI corrigem o erro em um tempo de subida semelhante. Mesmo o controlador fuzzy tendo sido sintonizado manualmente conseguiu ter um tempo de subida idêntico ao do controlador PI, porém sem sobressinal, demonstra a sua capacidade de desassociar a frequência natural e o amortecimento equivalentes, algo que o controlador PI não consegue. 


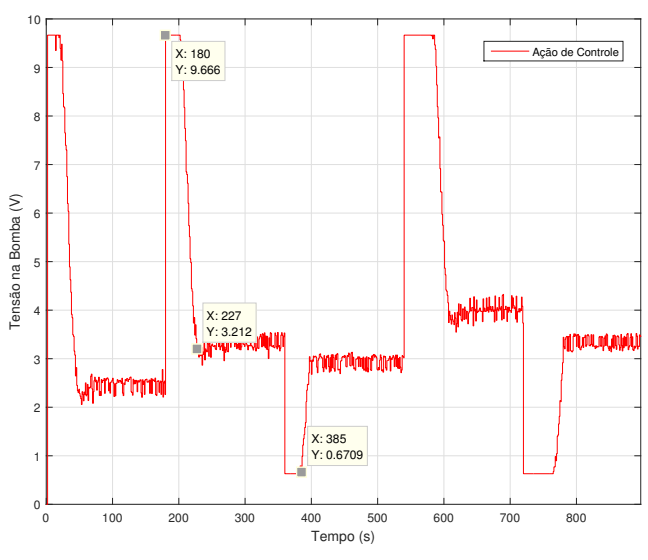

Figura 12. Ação do Controlador PI.

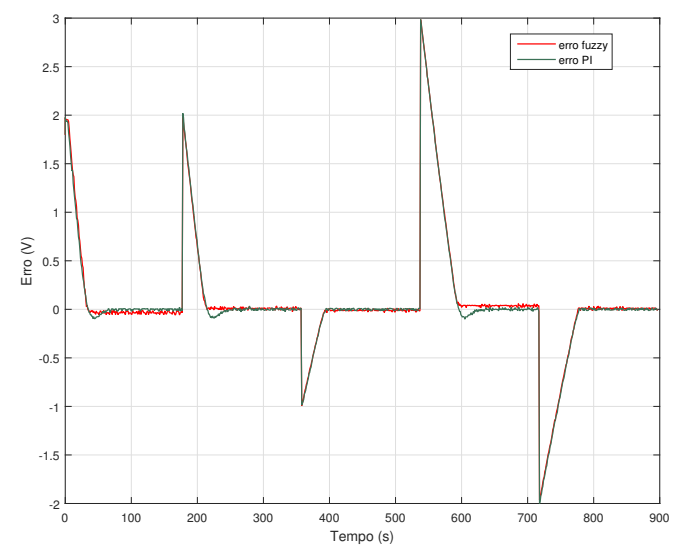

Figura 13. Erro do Controlador Fuzzy e Pi.

\section{CONCLUSÃO}

O controlador fuzzy desenvolvido neste trabalho teve toda sua sintonia realizada de forma manual, possuindo 8 regras relacionando as variáveis linguísticas associadas ao erro e a variação do nível de tanque. O PI utilizado para comparação com o controle proposto foi sintonizado por método de alocação de polos.

O controle fuzzy apresentado nesse trabalho foi eficaz, controlando o nível do tanque em questão. O controle PI foi mais complexo de ser implementado em relação ao fuzzy, devido o fato de ser necessário encontrar o modelo matemático do sistema de nível do tanque. Na aplicação do controlador fuzzy foi necessário apenas conhecer o sistema e como ele se comporta, onde todas as funções de pertinências e regras implementadas foram baseadas na experiencia do operador.

O controle fuzzy, apesar que sua sintonia ter sido manual, mostrou-se mais eficiente que o controlador PI por não apresentar overshooot. O ruído encontrado na ação de controle fuzzy pode ser futuramente reduzido adicionandose mais funções de pertinência nas entradas erro e rate, e fazendo uma sintonia automática de suas funções de pertinências, aumentando assim sua eficiência.

\section{AGRADECIMENTOS}

Os autores agradecem ao programa de pós graduação em engenharia elétrica da UFPI, ao grupo de pesquisa em automação e sistemas inteligentes (GRASI), e a CAPES pelo apoio financeiro.

\section{REFERÊNCIAS}

Bhandare, D.S. and Kulkarni, N. (2015). Performances evaluation and comparison of pid controller and fuzzy logic controller for process liquid level control. In Control, Automation and Systems (ICCAS), 2015 15th International Conference on, 1347-1352. IEEE.

Branco, H.M.G.C. (2009). Uma estratégia para a detecção e classificação de transitórios em transformadores de potência pela utilização da transformada Wavelet e da lógica Fuzzy. Ph.D. thesis, Universidade de São Paulo.

Franzini, T.D., Pinheiro, R.M., Carneiro, M.R., and Martins, C. (2013). Aplicações de técnicas de controle no matlab/simulink com comunicação via opc em uma planta didática hart. XLI Congresso Brasileiro de Educação em Engenharia, Gramado, RS.

Gomide, F., Gudwin, R.R., and Tanscheit, R. (1995). Conceitos fundamentais da teoria de conjuntos fuzzy, lógica fuzzy e aplicações. In Proc. 6 th IFSA CongressTutorials, 1-38.

Guarnizo, J. and Avendano, J. (2017). Liquid level system as a pedagogical tool to teach fuzzy control. In Electronics, Communications and Computers (CONIELECOMP), 2017 International Conference on, 1-5. IEEE.

Helmich, J. (????). Adiro. Manual MPS-PA Compact Workstation, volume $=12$, year $=2008$.

Kannan, R., Raj, P.A., and Poongodi, P. (2010). Design of fuzzy immune pid controller for liquid level control system. In 2010 Second International Conference on Computer and Network Technology, 566-570. IEEE.

Lamkhade, P.N., Parvat, B., and Kadu, C. (2015). Design and implementation of fuzzy logic controller for level control. In 2015 International Conference on Energy Systems and Applications, 475-479. IEEE.

Parvat, B. and Patre, B. (2015). Second order sliding mode controller for second order process with delay time. In 2015 International Conference on Industrial Instrumentation and Control (ICIC), 280-284. IEEE.

Prusty, S.B., Pati, U.C., and Mahapatra, K. (2014). Implementation of fuzzy-pid controller to liquid level system using labview. In Proceedings of The 2014 International Conference on Control, Instrumentation, Energy and Communication (CIEC), 36-40. IEEE.

Sandri, S. and Correa, C. (1999). Lógica nebulosa. Instituto Tecnológico da Aeronáutica-ITA, V Escola de Redes Neurais, pp. C073-c090, São José dos Campos.

Shaw, I.S. (2007). Controle e modelagem fuzzy. Blucher: FAPESP, São Paulo, BRA.

Silva, P.C., Matos, D.S., Cavalca, M.S., and Nied, A. (2014). Projeto de um controlador adaptativo aplicado à estação mps-pa da festo no sistema de pressão. $X X$ Congresso Brasileiro de Automática, Belo Horizonte, $M G$.

Zadeh, L.A. (1965). Fuzzy sets. Information and control, $8(3), 338-353$. 